\title{
Theoretical Study of the Effect of Ionospheric Return Currents on the Electron Temperature
}

\author{
R. W. SChunk, J. J. SoJKA, AND M. D. Bowline \\ Center for Atmospheric and Space Sciences, Utah State University, Logan
}

\begin{abstract}
An electron heat flow can occur in a partially ionized plasma in response to either an electron temperature gradient (thermal conduction) or an electron current (thermoelectric heat flow). The former process has been extensively studied, while the latter process has received relatively little attention. Therefore a time-dependent three-dimensional model of the high-latitude ionosphere was used to study the effect of field-aligned ionospheric return currents on auroral electron temperatures for different seasonal and solar cycle conditions as well as for different upper boundary heat fluxes. The results of this study lead to the following conclusions: (1) The average, large-scale, return current densities, which are a few microamps per square meter, are too small to affect auroral electron temperatures. (2) Current densities greater than about $10^{-5} \mathrm{~A} \mathrm{~m}^{-2}$ are needed for thermoelectric heat flow to be important. (3) The thermoelectric effect displays a marked solar cycle and seasonal dependence. (4) Thermoelectric heat transport corresponds to an upward flow of electron energy. (5) This energy flow can be either a source or sink of electron energy, depending on the altitude and geophysical conditions. (6) Thermoelectric heat transport is typically a sink above $300 \mathrm{~km}$ and acts to lower ambient electron temperatures by as much as $2000 \mathrm{~K}$ for field-aligned return current densities of the order of $5 \times 10^{-5} \mathrm{~A} \mathrm{~m}^{-2}$. For this case, the electron temperature decreases with altitude above 300 $\mathrm{km}$ with a gradient that can exceed $1 \mathrm{~K} \mathrm{~km}^{-1}$. Also, the electron temperature can drop below both the ion and neutral temperatures in the upper $F$ region owing to thermoelectric cooling. (7) A downward magnetospheric heat flux in combinations with an upward thermoelectric heat flux can produce steep positive electron temperature gradients in the topside ionosphere.
\end{abstract}

\section{INTRODUCTION}

It is well known that an electron heat flow can occur in a partially ionized plasma in response to either an electron temperature gradient or an electron current. The former process corresponds to thermal conduction, while the latter process is known as thermoelectric heat transport [cf. Chapman and Cowling, 1970; Spitzer, 1956; Shkarofsky, 1961]. With regard to ionospheric applications the first suggestion that thermoelectric heat transport could affect electron temperatures in the auroral oval was made by Schunk and Walker [1970]. On the basis of a simple comparison of thermoelectric and thermal conduction terms these authors concluded that auroral electron temperatures could be significantly affected by thermoelectric heat transport if the field-aligned current density is greater than about $10^{-5} \mathrm{~A} \mathrm{~m}^{-2}$.

After this initial suggestion, Rees et al. [1971] conducted a more quantitative study to determine the magnitude of the thermoelectric effect. Specifically, these authors solved the coupled electron and ion energy equations with allowance for heat transport due to a field-aligned current. In their model aurora they assumed that a precipitating flux of auroral electrons produces a flow of reverse current that is equal in magnitude to the current carried by the precipitating auroral electrons at all altitudes. This reverse current was of the order of $2.5 \times 10^{-5}$ $\mathrm{A} \mathrm{m}^{-2}$. However, because the upper boundary heat flux was unknown, they considered two cases; zero net heat flux and zero conducted heat flux through the upper boundary. From their study, Rees et al. [1971] found that thermoelectric heat transport acts to cool the ambient electron gas by as much as $1000 \mathrm{~K}$ above $700 \mathrm{~km}$. They also found that thermoelectric heat trans-

Copyright 1987 by the American Geophysical Union.

Paper number 6A8865.

0148-0227/87/006A-8865\$05.00 port produces an upward energy flow that approached $10^{10} \mathrm{eV}$ $\mathrm{cm}^{-2} \mathrm{~s}^{-1}$ at high altitudes for the value of the field-aligned current they adopted.

The effect of ionospheric return currents on auroral electron temperatures was also studied by Ganguli [1986]. In this study the auroral field line plasma was modeled with a one-dimensional, multimoment, multifluid set of transport equations, with the emphasis of the study devoted to understanding magnetosphere-ionosphere coupling phenomena at high altitudes $(>$ $1500 \mathrm{~km}$ ). This study elucidated some very interesting dynamical properties of the auroral electron temperature in the highaltitude collisionless regime, including the development of a temperature anisotropy in response to return current onset. However, this work is not directly related to the above studies that pertain to ionospheric altitudes.

To our knowledge, there have been no further studies of the effect of thermoelectric heat transport on $F$ region auroral electron temperatures. However, Rees et al. [1971] considered only one field-aligned current configuration and only one geophysical situation. Also, at the time of the Rees et al. [1971] study the nature of the Birkeland current system was not well established. Since this time, a large amount of information has been gathered on the Birkeland current system, and presently, there is considerable interest in the electron temperature variation at high latitudes [Kofman and Wickwar, 1984; Wickwar and Kofman, 1984; Curtis et al., 1985]. Therefore we have used our high-latitude ionosphere model, which has been extended recently to include the electron energy equation [Schunk et al., 1986], to study the effect of ionospheric return currents on auroral electron temperatures for a range of conditions. The thermoelectric effect was studied for summer and winter conditions at both solar maximum and solar minimum. For each geophysical situation, several field-aligned current values were used in combination with different upper boundary heat fluxes. 


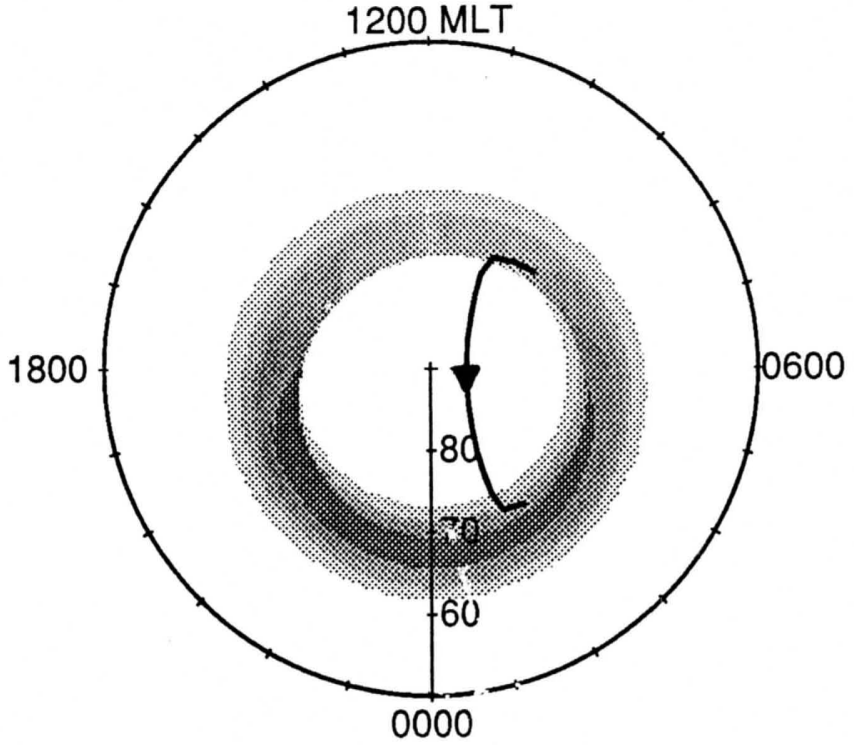

Fig. 1a. Shaded contours of the precipitating auroral electron energy flux in a magnetic latitude, MLT reference frame. The energy flux varies from a low of $0.1 \mathrm{erg} \mathrm{cm}^{-2} \mathrm{~s}^{-1}$ (lightest shade) to a high of $7 \mathrm{ergs} \mathrm{cm}^{-2} \mathrm{~s}^{-1}$ (darkest shade). The solid line is part of our adopted convection trajectory. The electron energy flux at the end of the trajectory is $0.44 \mathrm{erg}$ $\mathrm{cm}^{-2} \mathrm{~s}^{-1}$.

\section{IONOSPHERE MODEL}

The ionospheric model was initially developed as a midlatitude, multi-ion $\left(\mathrm{NO}^{+}, \mathrm{O}_{2}^{+}, \mathrm{N}_{2}^{+}\right.$, and $\mathrm{O}^{+}$) model by Schunk and Walker [1973]. The time-dependent ion continuity and momentum equations were solved as a function of altitude for a corotating plasma flux tube including diurnal variations and all relevant $E$ and $F$ region processes. This model was extended to include high-latitude effects due to convection electric fields and particle precipitation by Schunk et al. [1975, 1976]. A simplified ion energy equation was also added, which was based on the assumption that local heating and cooling processes dominate (valid below $500 \mathrm{~km}$ ). Flux tubes of plasma were followed as they moved in response to convection electric fields. A further extension of the model to include the minor ions $\mathrm{N}^{+}$and $\mathrm{He}^{+}$, an updated photochemical scheme, and the mass spectrometer/ incoherent scatter (MSIS) atmospheric model is described by Schunk and Raitt [1980].

The addition of plasma convection and particle precipitation models is described by Sojka et al. [1981a, b]). More recently, the ionospheric model has been extended by Schunk and Sojka [1982] to include ion thermal conduction and diffusion-thermal heat flow, so that the ion temperature is now rigorously calculated at all altitudes between 120 and $1000 \mathrm{~km}$. The adopted ion energy equation and conductivities are those given by Conrad and Schunk [1979]. Also, the electron energy equation has been included recently by Schunk et al. [1986], and consequently, the electron temperature is now rigorously calculated at all altitudes. The electron energy equation and the heating and cooling rates were taken from Schunk and Nagy [1978], and the conductivities were taken from Schunk and Walker [1970].

With the high-latitude model, flux tubes of plasma are followed as they convect through a moving neutral atmosphere. Altitude profiles of the ion and electron temperatures and the $\mathrm{NO}^{+}, \mathrm{O}_{2}^{+}, \mathrm{N}_{2}^{+}, \mathrm{N}^{+}, \mathrm{O}^{+}$, and $\mathrm{He}^{+}$densities are obtained by solving the appropriate continuity, momentum, and energy equations, including all of the high-latitude processes thought to be impor- tant. The equations are typically solved over the altitude range from 120 to $800 \mathrm{~km}$, with boundary conditions specified at the lower and upper ends of the flux tubes. For the densities, chemical equilibrium is assumed at $120 \mathrm{~km}$ and no escape flux is assumed at $800 \mathrm{~km}$. For the temperatures, local thermal coupling is assumed at $120 \mathrm{~km}$ and a specified heat flux is assumed at $800 \mathrm{~km}$. For this study the ion heat flux through the upper boundary was taken to be zero and the electron heat flux was varied.

In this study we focus attention on the electron thermal structure in the presence of ionospheric return currents. However, since the appropriate electron energy equation, thermal conductivities, and heating and cooling processes have been extensively discussed in the literature [Schunk and Walker, 1970; Rees et al., 1971; Schunk and Nagy, 1978; Schunk et al., 1986], we will not repeat them here. For this study the important thing to note is that in the presence of a thermoelectric heat flow the total electron heat flow is given by

$$
\mathbf{q}_{e}=-\beta_{e} \mathbf{J}-K^{e} \nabla T_{e}
$$

where $\mathbf{q}_{e}$ is the total electron heat flow, $\mathbf{J}$ is the field-aligned ionospheric return current, $\nabla T_{e}$ is the electron temperature gradient, $\beta_{e}$ is the thermoelectric coefficient, and $K^{e}$ is the electron thermal conductivity. Note that equation (1) applies along the flux tube and that it only applies to the thermal electrons. Precipitating auroral electrons require a separate formulation (see next section).

It is apparent from equation (1) that heat will flow along a flux tube in response to both a field-aligned current and a temperature gradient. In our study we considered several values for the return current in combination with different values for the total heat flux at the upper boundary. This procedure was adopted because, at present, the relationship between the return current and the upper boundary heat flux, if any, is not known.

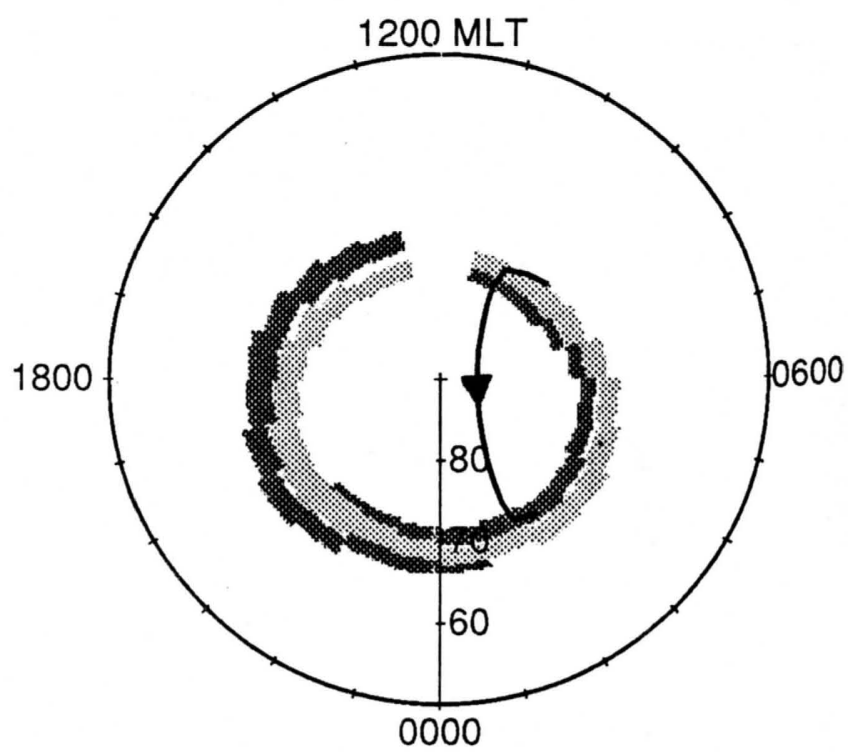

Fig. 1b. Birkeland currents linking the northern high-latitude ionosphere with the magnetosphere. The dark shading shows the ionospheric return current region (upward thermal electrons), and the light shading shows the upward current region (downward precipitating electrons). The solid line shows our adopted convection trajectory, which ends in the return current region. 
SOLAR MINIMUM

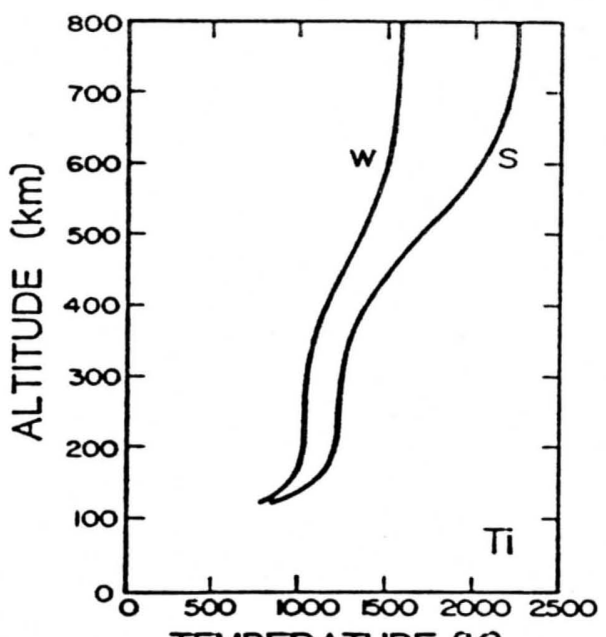

TEMPERATURE (K)
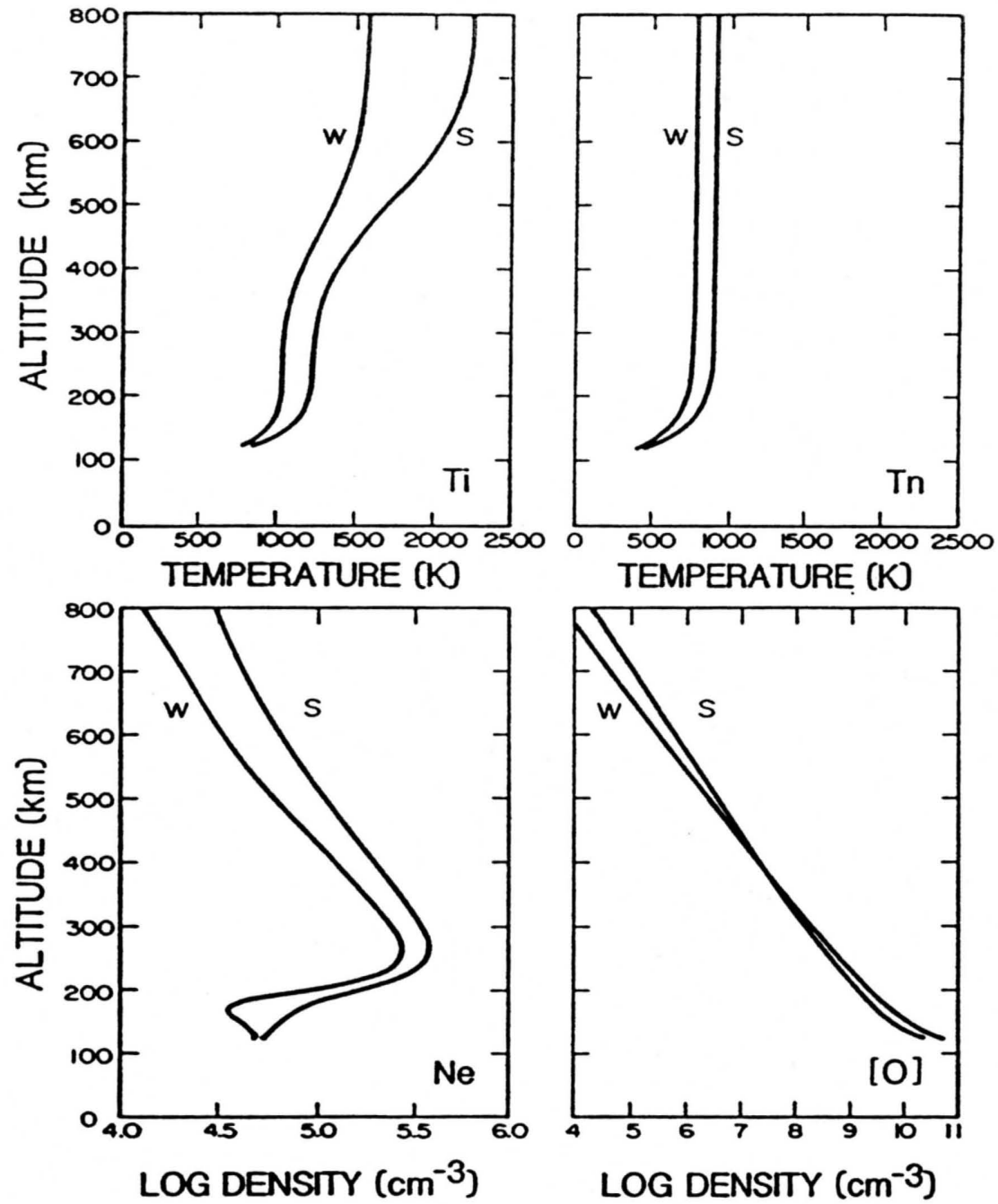

Fig. 2. Altitude profiles of $T_{i}, T_{n}, N_{e}$ and [O] for summer (S) and winter (W) conditions at solar minimum. These profiles pertain to the conditions at the end of our adopted convection trajectory.

\section{MOdEL INPUTS}

There are several inputs that are needed for our high-latitude ionospheric model, including the neutral atmosphere, thermospheric wind, plasma convection model, auroral oval, and a pattern for the ionospheric return current. For the neutral atmosphere we adopted the MSIS model [Hedin et al., 1977] with 1979 day 185 representing summer, day 360 representing winter, $F_{10.7}=220 \times 10^{-22} \mathrm{~W} \mathrm{~m}^{-2} \mathrm{~Hz}^{-1}$ for solar maximum, $F_{10.7}=70 \times 10^{-22} \mathrm{~W} \mathrm{~m}^{-2} \mathrm{~Hz}^{-1}$ for solar minimum, and $A p=20$ for all four cases. For the thermospheric wind we adopted a simple pattern with an antisunward flow over the polar cap at a speed of about $200 \mathrm{~m} \mathrm{~s}^{-1}$. This wind pattern has been used in several of our previous studies and is described by Sojka et al. $[1981 a, b]$.

Electron precipitation in the auroral oval acts as a plasma production source, a source of bulk heating for the thermal electrons, and a source of heat that flows through our upper boundary. For our auroral oval we adopted the empirical model developed by Spiro et al. [1982] and used an $A_{e}$ value of 260 . Figure $1 a$ shows contours of the auroral electron energy flux in ergs per square centimeter per second in an MLT magnetic latitude reference frame. The adopted oval is approximately $10^{\circ}$ wide, with the strongest precipitation occurring in the night sector where the energy flux reaches $7 \mathrm{ergs} \mathrm{cm}^{-2} \mathrm{~s}^{-1}$. The procedure for calculating the ionization rate and the thermal electron bulk heating rate from the auroral electron energy flux is described by Schunk et al. [1986].

Also shown in Figure $1 a$ is part of our adopted convection trajectory. This trajectory was taken from a symmetric, two-cell convection pattern of the Volland [1978] type. The pattern corresponds to moderate magnetic activity with $K p \sim 4$ and a total cross-polar cap potential of $76 \mathrm{kV}$. In the polar cap the electric field is about $23 \mathrm{mV} \mathrm{m}^{-1}$, and the corresponding antisunward convection speed is about $450 \mathrm{~m} \mathrm{~s}^{-1}$. For most of our calculations, flux tubes of plasma were followed along this trajectory starting on the dayside near 0900 MLT and ending on the nightside where the trajectory segment ends, which is in the return current region. Below, we list the important model input parameters at this location.

The final input needed for our study is the distribution of field-aligned or Birkeland currents. A considerable effort has been directed toward elucidating the characteristics of these currents during the last 20 years, and spatial distributions are 


\section{SOLAR MAXIMUM}
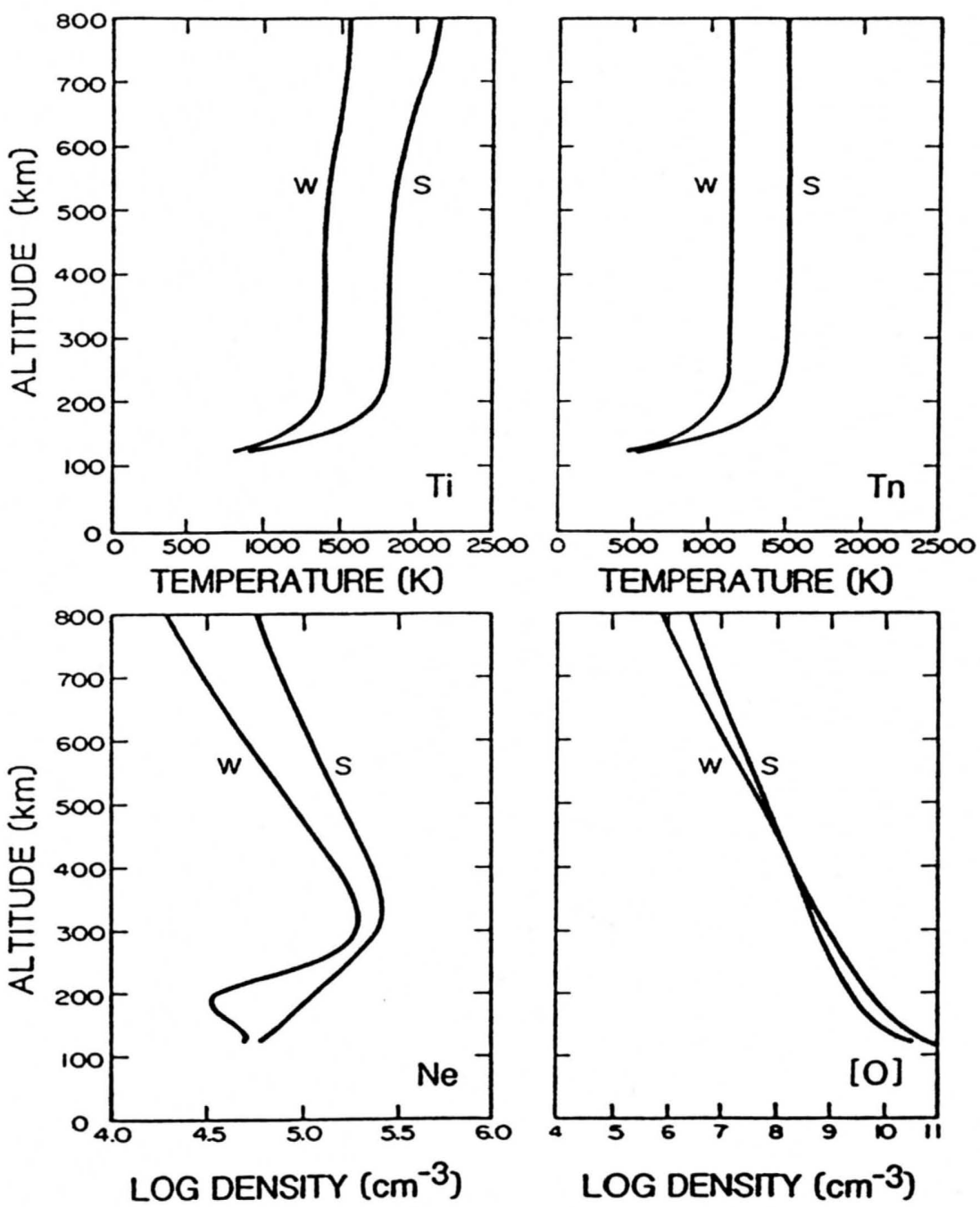

Fig. 3. Altitude profiles of $T_{i}, T_{n}, N_{e}$ and [O] for summer (S) and winter (W) conditions at solar maximum. These profiles pertain to the conditions at the end of our adopted convection trajectory.

readily available [Zmuda et al., 1966; Zmuda and Armstrong, 1974; Sugiura, 1975; Iijima and Potemra, 1976]. For this study we adopted the Birkeland current pattern presented by Iijima and Potemra [1976]. These authors determined the spatial distribution and magnitudes of field-aligned currents at $800 \mathrm{~km}$ over the northern high-latitude region from Triad magnetometer data recorded at College, Alaska. The Birkeland current distribution was determined from 1300 satellite passes during the 16-month period from July 1973 to October 1974.

Figure $1 b$ shows the average distribution of the large-scale field-aligned currents deduced by Iijima and Potemra [1976] for geomagnetic conditions characterized by $2^{-} \leq K p \leq 4^{+}$(Figure $1 b$ is a replot of Iijima and Potemra's Figure 2). The fieldaligned currents are concentrated in two principal areas encircling the geomagnetic pole. The dark shading shows the ionospheric return current region (upward thermal electrons), and the light shading shows the upward current region (downward precipitating electrons). Typically, the magnitudes of the average field-aligned currents are of the order of a few microamps per square meter.

It is important to note that our theoretical formulation only takes into account the effect of ionospheric return currents, that is, the motion of the upward thermal electrons. The downward precipitating electrons are a magnetospheric population that must be treated separately. Unfortunately, at the present time it is not clear how to treat this population. At $F$ region altitudes the precipitating auroral electrons are collisionless, and a collision-dominated formulation similar to the one we used for the ionospheric thermal electrons is not appropriate. Kinetic solutions can be constructed for auroral electrons [cf. Knight, 1973], but only for steady state conditions. Also, further complications arise because the precipitating auroral electrons excite waves all along the flux tube, and it is not known how these waves affect the transport properties of the plasma. Initial studies of such effects have been undertaken by Mitchell and Palmadesso [1984] using anomalous resistivity coefficients to describe the wave-particle interactions, but further work needs to be done before definitive conclusions can be drawn as to how to treat the precipitating auroral electrons. Therefore at the present time the thermoelectric effect in the upward current region (Figure $1 b$ ) due to precipitating electrons cannot be rigorously studied. However, the effect may not be important because the precipitating auroral electrons produce secondaries owing to collisions with the neutrals, and these secondary elec- 

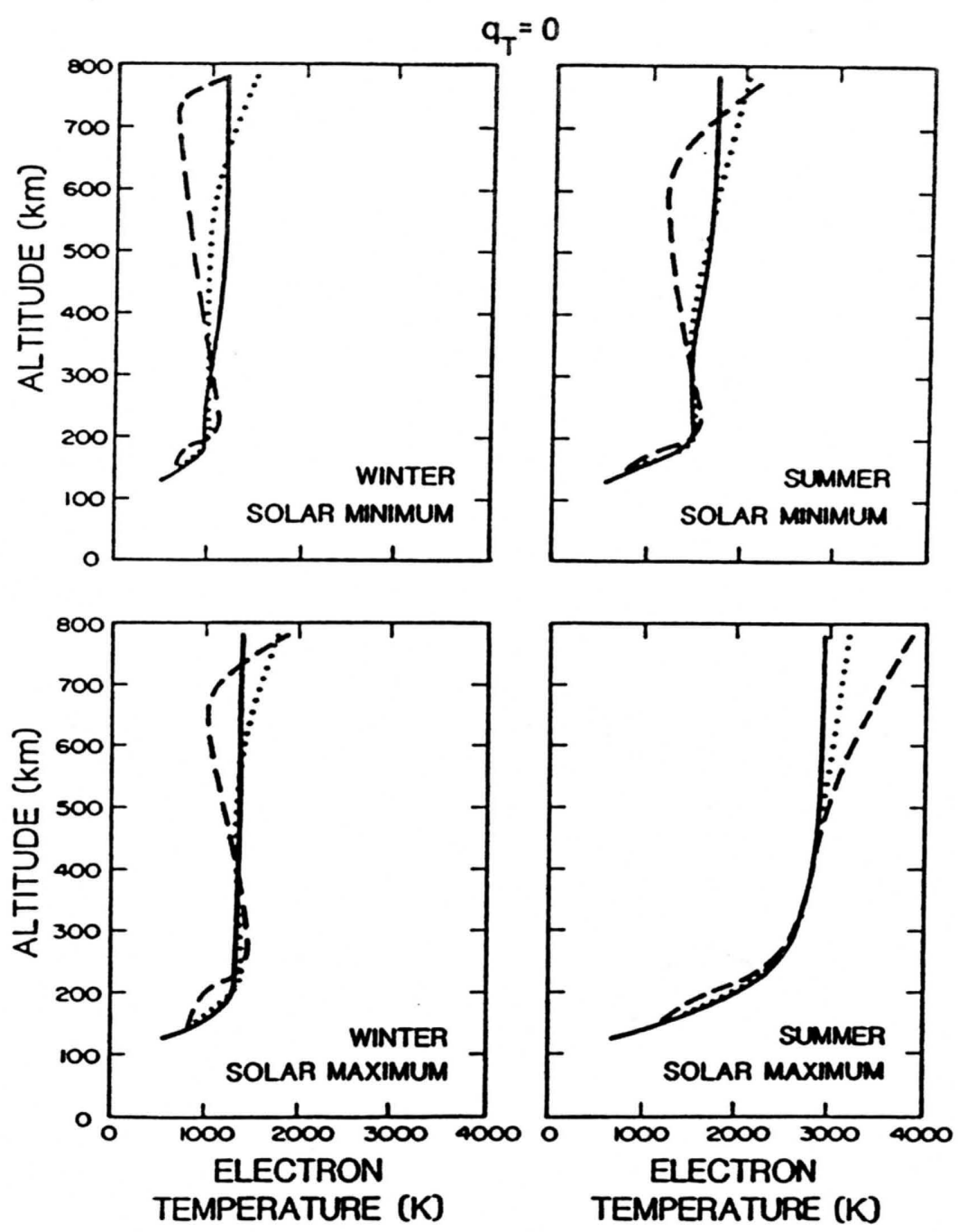

Fig. 4. Electron temperature profiles for three values of the field-aligned current for summer and winter conditions at both solar maximum and solar minimum. The field-aligned current values are 0 (solid curves), $-1 \times 10^{-5}$ (dotted curves), and $-5 \times$ $10^{-5}$ (dashed curves) $\mathrm{A} \mathrm{m}^{-2}$. The profiles were calculated at the end of our adopted convection trajectory with no upper boundary electron heat flux.

trons provide a significant source of bulk heating for the ionospheric electrons that could dominate a thermoelectric effect due to the auroral electrons.

As noted above, we will present results at the end of our adopted convection trajectory, and therefore it is convenient to summarize some of the model input parameters at this location. The end of the trajectory is located at $69.9^{\circ}$ latitude and 2.35 MLT, the auroral electron energy flux is $0.44 \mathrm{erg} \mathrm{cm}^{-2} \mathrm{~s}^{-1}$, the convection electric field is $32 \mathrm{mV} \mathrm{m}^{-1}$, and the average value of the ionospheric return current is $-1 \mu \mathrm{A} / \mathrm{m}^{2}$.

\section{EFFECT OF RETURN CURRENTS ON ELECTRON TEMPERATURES}

To study the effect of ionospheric return currents on the electron temperature, we calculated ion and electron densities and temperatures self-consistently for a flux tube of plasma that followed the trajectory segment shown in Figure 1a. Daytime steady state profiles were first calculated at the start of the trajectory near $0900 \mathrm{MLT}$, and then the flux tube of plasma was followed as it moved along the trajectory and eventually entered the ionospheric return current region on the nightside. In the figures that follow we present the profiles obtained at the end of the trajectory segment.

Since the electron temperature is strongly affected by the neutral atomic oxygen density [O], the electron density $N_{e}$, the neutral temperature $T_{n}$, and the ion temperature $T_{i}$, it is useful to show these parameters at the end of the convection trajectory. Figure 2 shows altitude profiles of [O], $N_{e}, T_{n}$, and $T_{i}$ for summer and winter conditions at solar minimum, while Figure 3 shows the same parameters at solar maximum. The seasonal trend is the same for all four parameters at solar minimum and solar maximum, with larger values of $N_{e}, T_{i}$, and $T_{n}$ at all altitudes in summer than in winter and lower values of [O] in the $F$ region in summer than in winter. Note, however, that the variation of $N_{e}$ with altitude is different below the $F$ peak in winter than in summer. In winter the ionosphere at the end of the convection trajectory is in darkness, and hence the lower $F$ region decays, while in summer it is sunlit (solar zenith angle of $79^{\circ}$ ).

With regard to the solar cycle effect the general trend is for larger values of $T_{i}, T_{n}$, and [O] at solar maximum than at solar minimum. However, the variation of the electron density is more complicated. Near the $F$ region peak, $N_{e}$ is greater at solar 

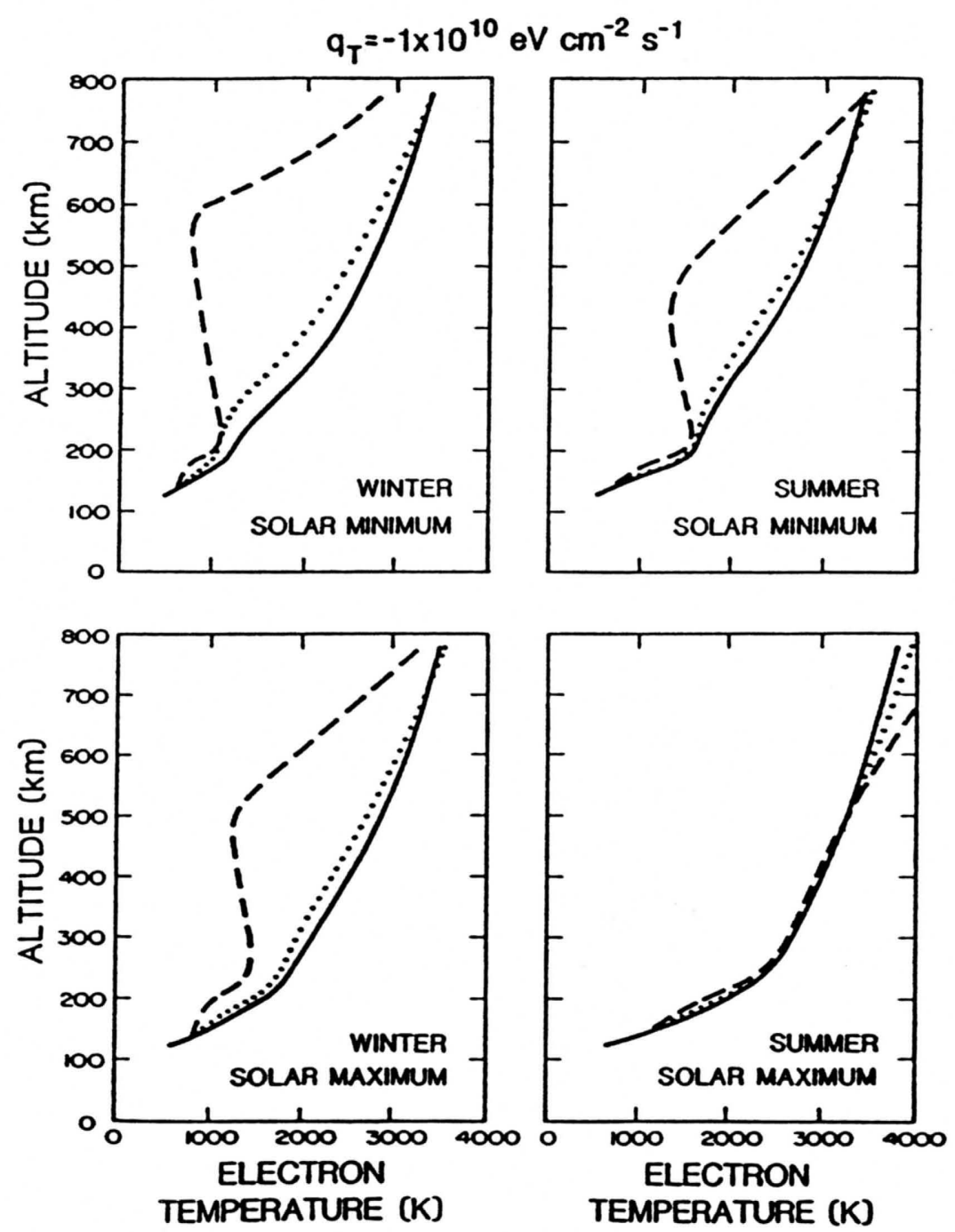

Fig. 5. Same as Figure 4 except that the upper boundary heat flux is $-1 \times 10^{10} \mathrm{eV} \mathrm{cm}^{-2} \mathrm{~s}^{-1}$.

minimum than at solar maximum, but the reverse is true at high altitudes owing to the higher temperatures and hence greater plasma scale heights at solar maximum.

A study of the effect of the ionospheric return currents shown in Figure $1 b$, which are of the order of a few microamps per square meter, indicates that the amplitudes of these "average" large-scale currents are too small to affect ambient electron temperatures via thermoelectric heat transport. This conclusion is based on a wide range of seasonal and solar cycle conditions as well as on the use of additional plasma convection trajectories not shown in Figure 1a. Therefore as far as large-scale ionospheric modeling is concerned, it is not necessary to include ionospheric return current effects in calculating $F$ region densities and temperatures.

Although ionospheric return current effects are not important for large-scale ionospheric modeling, they could be very significant at certain places and times. The problem with the Birkeland current patterns of the type shown in Figure $1 b$ is that a significant spatial and temporal averaging is used in constructing these "average" patterns. For example, the spatial distribution shown in Figure $1 b$ was obtained by averaging data over $1 / 2^{\circ}$ of latitude [cf. Iijima and Potemra, 1976]. However, recent data acquired by the Dynamic Explorer satellite indicate that the Birkeland currents are highly structured and that the current densities can be orders of magnitude greater at certain places and times than the average values shown in Figure $1 b(\mathrm{M}$. Sugiura, private communication, 1986). Therefore we considered larger values of the return current and studied their effect on the electron temperature for the full range of seasonal and solar cycle conditions described above. However, for these cases we did not calculate consistent ion densities and temperatures. Instead, we used the ion densities and temperatures shown in Figures 2 and 3, which pertain to the conditions at the end of our convection trajectory, as inputs to the electron energy equation and then obtained new electron temperature profiles for different return current densities as well as for different upper boundary heat fluxes. Since the time constant for electron temperature changes is only a few seconds and the time constant for ion density changes is tens of minutes, this simplification should not introduce appreciable errors because the flux tube of plasma will typically convect out of the return current region before the density can adjust to the new electron temperature distribution.

Figure 4 shows electron temperature profiles for three values of the field-aligned return current for summer and winter conditions at both solar maximum and solar minimum. The field- 


\section{UPWARD HEAT FLOW}
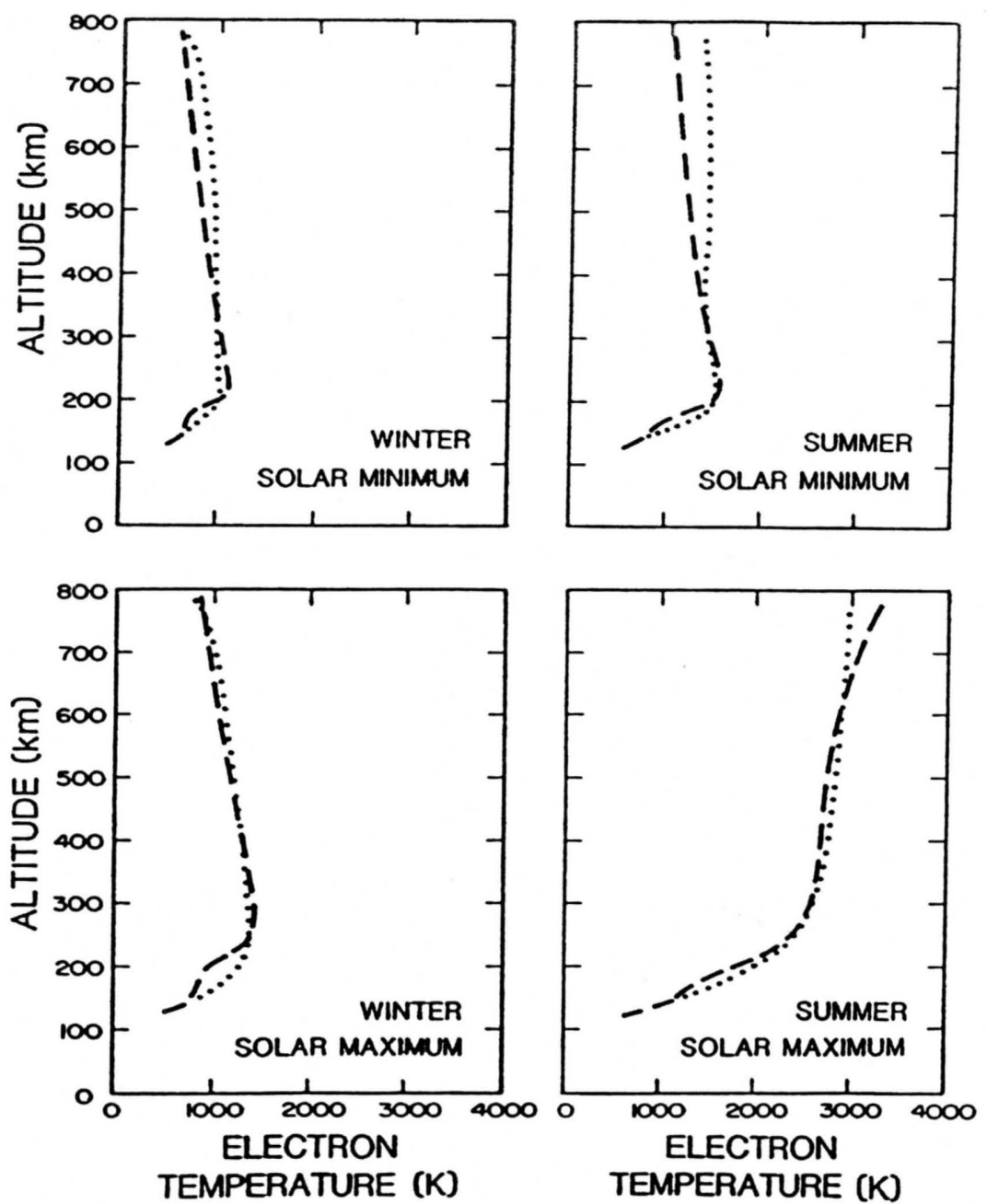

Fig. 6. Same as Figure 4 except for the upper boundary heat flux. The boundary electron heat fluxes are $+2 \times 10^{9}$ (dotted curves) and $+7 \times 10^{9}$ (dashed curves) $\mathrm{eV} \mathrm{cm}^{-2} \mathrm{~s}^{-1}$.

aligned currents are 0 (solid curves), $-1 \times 10^{-5}$ (dotted curves), and $-5 \times 10^{-5}$ (dashed curves) $\mathrm{A} \mathrm{m}^{-2}$. The profiles were calculated with the total heat flow through the upper boundary set to zero. The solid curves correspond to the typical case of no field-aligned current and no heat flow through the upper boundary. Consequently, the electron temperature is constant at high altitudes owing to the dominance of thermal conduction. For this case, the variation of $T_{e}$ with season and solar cycle is simply a consequence of the variations of [O], $N_{e}, T_{i}$, and $T_{n}$ with these parameters. Since this variation has been discussed extensively in the literature, we will not discuss it here.

For all the cases shown in Figure 4, thermoelectric heat transport corresponds to an upward flow of energy at all altitudes, which is in harmony with the one case considered by Rees et al. [1971]. However, Rees et al. [1971] found thermoelectric heat transport to be a sink at all altitudes, but we find that it can be a source or sink depending on the shape of the electron density profile. For example, if we consider the solar minimum winter case with $J_{\|}=-5 \times 10^{-5} \mathrm{~A} \mathrm{~m}^{-2}$, thermoelectric heat transport is a sink below $200 \mathrm{~km}$, a source between 200 and 280 $\mathrm{km}$, and a sink above $280 \mathrm{~km}$. The effect of an increased magnitude of the return current is merely to enhance this result. Rees et al. [1971] did not get this result because their adopted $N_{e}$ profile decreased with altitude all the way from $115 \mathrm{~km}$ to the topside ionosphere.

Note that above $280 \mathrm{~km}$ for the $J_{\|}=-5 \times 10^{-5} \mathrm{~A} \mathrm{~m}^{-2}$ curve the electron temperature decreases with altitude up to $750 \mathrm{~km}$ with a gradient of about $1 \mathrm{~K} \mathrm{~km}^{-1}$ because of the cooling associated with thermoelectric heat transport. At $720 \mathrm{~km}, T_{e}=680 \mathrm{~K}$, which is lower than $T_{n}(\sim 750 \mathrm{~K})$. Note also that $T_{e}$ is much lower than $T_{i}$, which is $1500 \mathrm{~K}$ at $720 \mathrm{~km}$. Above about $720 \mathrm{~km}$ there is an abrupt change in the $T_{e}$ gradient. This occurs because for the cases shown in Figure 4 we assumed that the total heat flux through the upper boundary was zero, and therefore a downward thermal conduction flux from the magnetosphere must balance the upward thermoelectric heat flux from the ionosphere at our upper boundary.

The solar minimum summer and solar maximum winter cases are qualitatively similar to the solar minimum winter case discussed above, but for solar maximum summer the results are different. Basically, thermoelectric heat transport is much less effective for solar maximum summer than for the other cases. This results because $T_{i}, T_{n}$, and $h_{m} F_{2}$ are high for solar maximum summer, and consequently, local heating and cooling processes dominate to a much higher altitude $(\sim 410 \mathrm{~km})$ than for the other cases. 

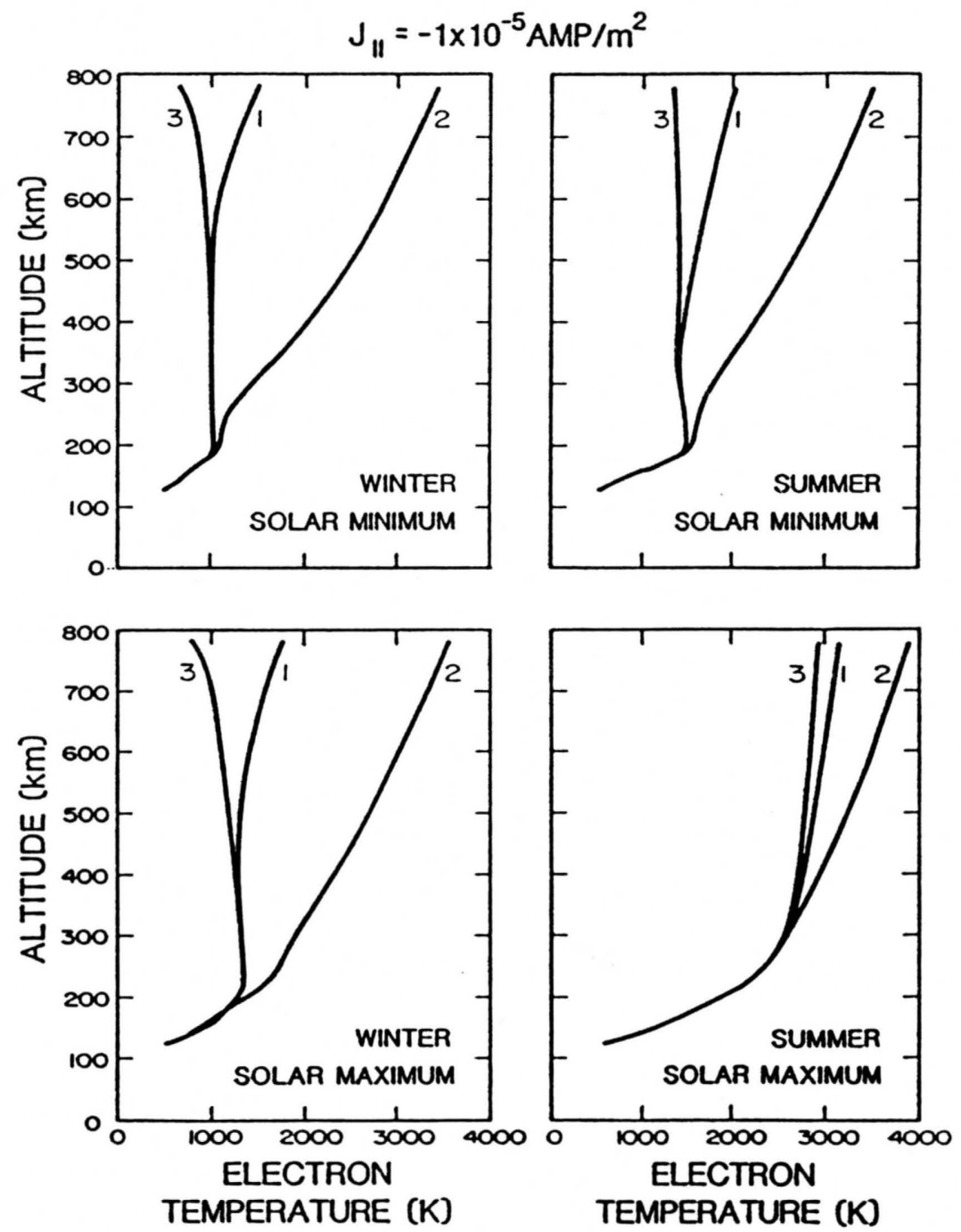

Fig. 7. Electron temperature profiles for different upper boundary heat fluxes for summer and winter conditions at both solar maximum and solar minimum. The field-aligned current is $-1 \times 10^{-5} \mathrm{~A} \mathrm{~m}^{-2}$, and the upper boundary heat fluxes are 0 (curve 1), $-1 \times 10^{10}$ (curve 2), and $+2 \times 10^{9}$ (curve 3 ) $\mathrm{eV} \mathrm{cm}^{-2} \mathrm{~s}^{-1}$. The profiles were calculated at the end of our convection trajectory.

Although it is possible for a downward magnetospheric heat flux to exactly cancel the upward thermoelectric heat flux at 800 $\mathrm{km}$, it is also possible that they will not exactly balance. Therefore we considered cases where the "net" heat flux through 800 $\mathrm{km}, q_{T}$, was both downward and upward. Figure 5 shows the results for a net downward heat flux of $-1 \times 10^{10} \mathrm{eV} \mathrm{cm}^{-2} \mathrm{~s}^{-1}$ at $800 \mathrm{~km}$. Other than this change, the seasonal, solar cycle, and return current conditions are the same as for Figure 4. With no return current (solid curves) the effect of a downward magnetospheric heat flux is to cause $T_{\mathrm{e}}$ to increase with altitude all the way to the top boundary. The inclusion of a return current for each of the geophysical cases produces a result that is qualitatively similar to that shown in Figure 4 for $q_{T}=0$. However, the magnitude of the thermoelectric cooling is greater for $q_{T}=-1 \times$ $10^{10} \mathrm{eV} \mathrm{cm}^{-2} \mathrm{~s}^{-1}$ than for $q_{T}=0$. For example, for the solar minimum winter case, thermoelectric cooling acts to reduce $T_{e}$ by $2000 \mathrm{~K}$ at $600 \mathrm{~km}$ when $J_{\|}=-5 \times 10^{-5} \mathrm{~A} \mathrm{~m}^{-2}$. The greater importance of heat transport processes when $q_{T}=-1 \times 10^{10} \mathrm{eV}$ $\mathrm{cm}^{-2} \mathrm{~s}^{-1}$ results from the higher electron temperatures coupled with the fact that $\beta_{e} \sim T_{e}$ and $K^{e} \sim T_{e}^{5 / 2}$ [cf. Schunk and Walker, 1970].
If the region above $800 \mathrm{~km}$ is isothermal, heat will not be conducted through our upper boundary, and consequently, the "net" heat flux through this boundary will be the upward thermoelectric heat flux induced by the ionospheric return current. Figure 6 shows the resulting $T_{e}$ profiles for upward heat fluxes at $800 \mathrm{~km}$. Except for this change, the seasonal, solar cycle, and return current conditions are the same as for Figure 4. A close comparison of Figures 6 and 4 indicates that only the temperatures above $300-400 \mathrm{~km}$ are affected by this change in $q_{T}$. Except for the solar maximum summer case, the dominance of thermoelectric heat transport all the way to the top boundary acts to produce a $T_{e}$ profile that decreases throughout the topside ionosphere. Note that for some of the geophysical cases shown in Figure 6, $T_{e}$ is less than both $T_{i}$ and $T_{n}$ at high altitudes.

It is convenient to replot the $T_{e}$ profiles in Figures 4-6 so that the effect of different upper boundary heat fluxes can be shown for a given value of the return current. Figure 7 shows the $T_{e}$ profiles for $J_{\|}=-1 \times 10^{-5} \mathrm{~A} \mathrm{~m}^{-2}$ and for three upper boundary heat fluxes $(0$, downward, and upward). For the two solar minimum cases and the solar maximum winter case, heat transport is important to altitudes as low as $150 \mathrm{~km}$, but for the solar 


$$
J_{\sharp}=-5 \times 10^{-5} \mathrm{AMP} / \mathrm{m}^{2}
$$
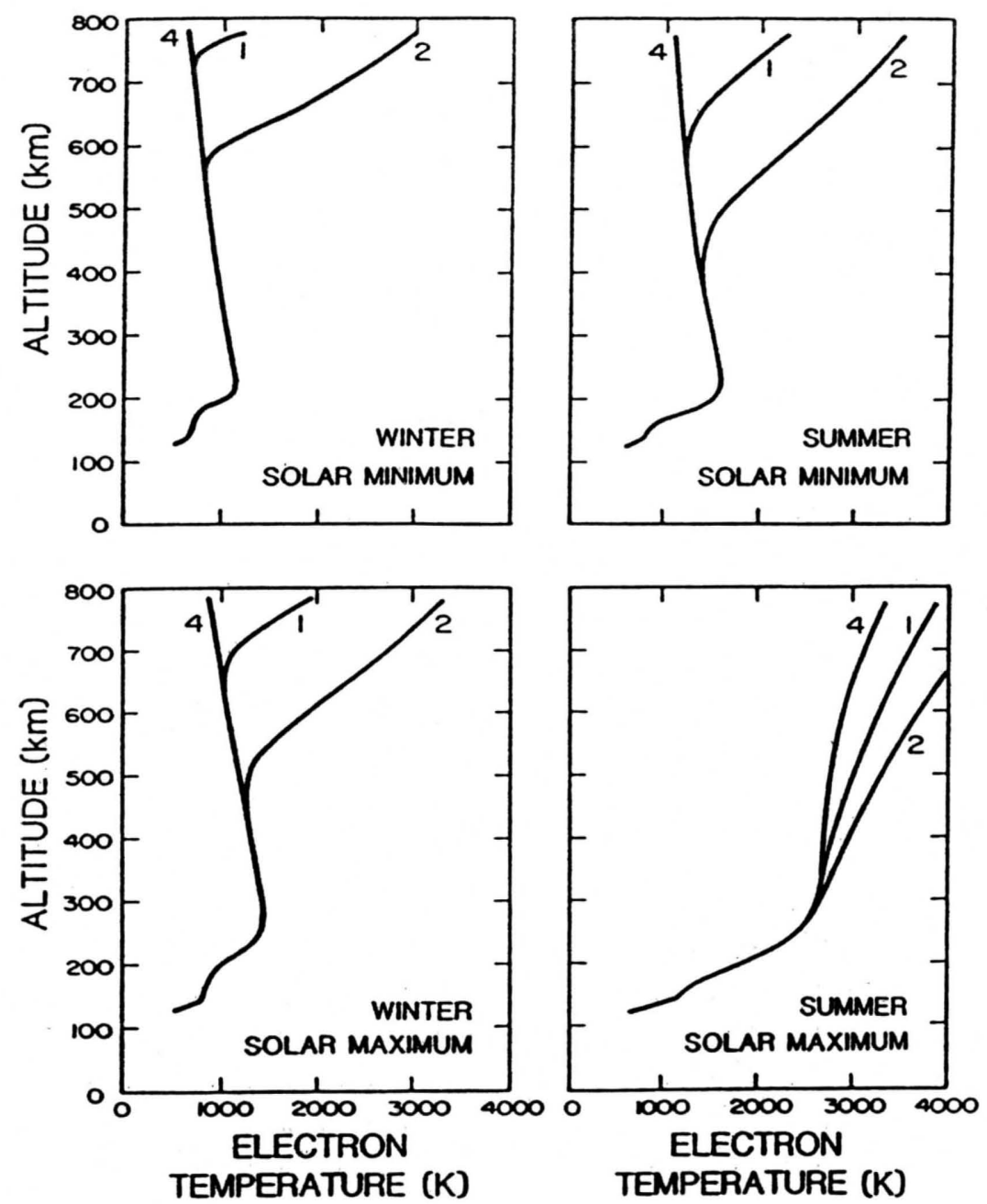

Fig. 8. Same as Figure 7 except that the field-aligned current is $-5 \times 10^{-5} \mathrm{~A} \mathrm{~m}^{-2}$, and the upper boundary heat fluxes are 0 (curve 1$),-1 \times 10^{10}$ (curve 2) and $+7 \times 10^{9}$ (curve 4) $\mathrm{eV} \mathrm{cm}^{-2} \mathrm{~s}^{-1}$.

maximum summer case, heat transport is important only above $300 \mathrm{~km}$. Also, for this latter case the effect of heat transport is much smaller than for the other geophysical cases.

Figure 8 shows the $T_{e}$ profiles for $J_{\|}=-5 \times 10^{-5} \mathrm{~A} \mathrm{~m}^{-2}$ for 0 , downward, and upward heat fluxes at $800 \mathrm{~km}$. With the larger value of the return current the electron cooling is much more dramatic, and a downward magnetospheric heat flux doesn't penetrate to low altitudes as it does for smaller values of $J_{\|}$ (compare curves labeled 2 in Figures 7 and 8). Also, with a larger return current the effect of a net downward heat flux at $800 \mathrm{~km}$ is to induce steep positive electron temperature gradients at high altitudes (curves 1 and 2 in Figure 8).

\section{SUMMARY}

We used a time-dependent three-dimensional model of the high-latitude ionosphere to study the effect of ionospheric return currents on auroral electron temperatures for a range of conditions. The thermoelectric heat transport that is associated with return currents was studied for summer and winter conditions at both solar maximum and solar minimum. For each geophysical situation, several field-aligned currents were used in combination with different upper boundary heat fluxes.
From our study we found the following:

1. The amplitudes of the return currents in the "average" large-scale current distributions presented by Iijima and Potemra [1976], which are of the order of a few microamps per square meter, are too small to affect auroral electron temperatures via thermoelectric heat transport.

2. Return current densities greater than about $10^{-5} \mathrm{~A} \mathrm{~m}^{-2}$ are needed in order for the associated thermoelectric heat transport to have an appreciable effect on electron temperatures.

3. The thermoelectric effect displays a marked solar cycle and seasonal dependence. It is important at solar minimum and in winter at solar maximum but not in summer at solar maximum. For the former geophysical situations, heat transport is important to altitudes as low as $150 \mathrm{~km}$, while for solar maximum summer, heat transport is only important above about 350 $\mathrm{km}$.

4. For all the cases we considered, thermoelectric heat transport corresponds to an upward flow of energy at all altitudes.

5. Thermoelectric heat transport can be either a source or sink of electron energy, depending on both the shape of the electron density profile and the magnitude of the adopted heat 
flux through the upper boundary. For $q_{T}=0$ and for the solar minimum and solar maximum winter cases, thermoelectric heat transport is a sink below $200 \mathrm{~km}$, a source between 200 and 280 $\mathrm{km}$, and a sink above $280 \mathrm{~km}$.

6. Thermoelectric heat transport is typically a sink above $300 \mathrm{~km}$ and acts to lower auroral electron temperatures by as much as $2000 \mathrm{~K}$ for $J_{\|}=-5 \times 10^{-5} \mathrm{~A} \mathrm{~m}^{-2}$. Because of the cooling the electron temperature decreases with altitude in the upper $F$ region with a gradient that can exceed $1 \mathrm{~K} \mathrm{~km}^{-1}$. Also, thermoelectric cooling can be sufficient to cause $T_{e}$ to drop below both $T_{i}$ and $T_{n}$ in the upper $F$ region.

7. The effect of a downward magnetospheric heat flow in combination with an upward thermoelectric heat flow is to cause steep positive electron temperature gradients in the topside ionosphere.

8. If there is no downward magnetospheric heat flow, $T_{e}$ can decrease with altitude from about $300 \mathrm{~km}$ to $800 \mathrm{~km}$ if the field-aligned return current density is sufficiently large.

Unfortunately, at the present time it is not possible to test the model predictions owing to the lack of a comprehensive data set. To verify the possible importance of thermoelectric heat transport, it is necessary to have observations in the return current region and to measure the field-aligned current density, the electron temperature as a function of altitude, the precipitating flux of soft auroral electrons, and the downward magnetospheric heat flux at high altitudes. It would also be useful to know the electron density and the ion and neutral temperatures as a function of altitude. The Sondrestrom and/or EISCAT incoherent scatter radars in combination with simultaneous satellite crossings could produce the required data. Preliminary indications of the possible occurrence of thermoelectric cooling would be an electron temperature profile that decreases with altitude above about $300 \mathrm{~km}$ or an unusually low electron temperature in the upper $F$ region.

Acknowledgments. This research was supported by NASA grant NAGW-77 and NSF grant ATM-8417880 to Utah State University.

The Editor thanks S. B. Ganguli and W. Hoegy for their assistance in evaluating this paper.

\section{REFERENCES}

Chapman, S., and T. G. Cowling, The Mathematical Theory of NonUniform Gases, Cambridge University Press, Cambridge, 1970.

Conrad, J. R., and R. W. Schunk, Diffusion and heat flow equations with allowance for large temperature differences between interacting species, J. Geophys. Res., 84, 811-822, 1979.

Curtis, S. A., W. R. Hoegy, L. H. Brace, and J. D. Winningham, Cusp altitudinal electron temperature gradient: Dynamics Explorer 2 implications for heating mechanisms, J. Geophys. Res., 90, 4415$4419,1985$.

Ganguli, S. B., Plasma transport in the auroral return current region, Ph.D. thesis, Boston College, 1986.

Hedin, A. E., C. A. Reber, G. P. Newton, N. W. Spencer, H. C. Brinton, H. G. Mayr, and W. E. Potter, A global thermospheric model based on mass spectrometer and incoherent scatter data, MSIS 2, Composition, J. Geophys. Res., 82, 2148, 1977.

Iijima, T., and T. A. Potemra, The amplitude distribution of fieldaligned currents at northern high latitudes observed by Triad, $J$. Geophys. Res., 81, 2165-2174, 1976.

Knight, S., Parallel electric fields, Planet. Space Sci., 21, 741-750, 1973.
Kofman, W., and V. B. Wickwar, Very high electron temperatures in the daytime $F$ region at Sondrestrom, Geophys. Res. Lett., 11, 919-922, 1984.

Mitchell, H. G., and P. J. Palmadesso, $\mathrm{O}^{+}$acceleration due to resistive momentum transfer in the auroral field line plasma, J. Geophys. Res., 89, 7573-7576, 1984.

Rees, M. H., R. A. Jones, and J. C. G. Walker, The influence of field-aligned currents on auroral electron temperature, Planet. Space Sci., 19, 313-325, 1971.

Schunk, R. W., and A. F. Nagy, Electron temperatures in the $F$-region of the ionosphere: Theory and observations, Rev. Geophys. Space Phys., 16, 355-399, 1978.

Schunk, R. W., and W. J. Raitt, Atomic nitrogen and oxygen ions in the daytime high-latitude $F$ region, J. Geophys. Res., 85, 1255-1272, 1980.

Schunk, R. W., and J. J. Sojka, Ion temperature variations in the daytime high-latitude $F$ region, $J$. Geophys. Res., 87, 5169-5183, 1982.

Schunk, R. W., and J. C. G. Walker, Transport properties of the ionospheric electron gas, Planet. Space Sci., 18, 1535-1550, 1970.

Schunk, R. W., and J. C. G. Walker, Theoretical ion densities in the lower ionosphere, Planet. Space Sci., 21, 1875-1896, 1973.

Schunk, R. W., W. J. Raitt, and P. M. Banks, Effect of electric fields on the daytime high-latitude $E$ and $F$ regions, J. Geophys. Res., 80, 3121-3130, 1975.

Schunk, R. W., P. M. Banks, and W. J. Raitt, Effects of electric fields and other processes upon the nighttime high latitude $F$ layer, $J$. Geophys. Res., 81, 3271-3282, 1976.

Schunk, R. W., J. J. Sojka, and M. D. Bowline, Theoretical study of the electron temperature in the high-latitude ionosphere for solar maximum and winter conditions, J. Geophys. Res., 91, 12,041-12,054, 1986.

Shkarofsky, I. P., Values of transport coefficients in a plasma for any degree of ionization based on a Maxwellian distribution, Can. J. Phys., 39, 1619-1703, 1961.

Sojka, J. J., W. J. Raitt, and R. W. Schunk, A theoretical study of the high-latitude winter $F$ region at solar minimum for low magnetic activity, J. Geophys. Res., 86, 609-621, 1981a.

Sojka, J. J., W. J. Raitt, and R. W. Schunk, Theoretical predictions for ion composition in the high-latitude winter $F$ region for solar minimum and low magnetic activity, J. Geophys. Res., 86, 2206-2216, $1981 b$.

Spiro, R. W., P. H. Reiff, and L. J. Maher, Precipitating electron energy flux and auroral zone conductances: An empirical model, J. Geophys. Res., 87, 8215-8227, 1982.

Spitzer, L., Physics of Fully Ionized Gases, Wiley-Interscience, New York, 1956.

Sugiura, M., Identifications of the polar cap boundary and the auroral belt in the high-latitude magnetosphere: A model for field-aligned currents, J. Geophys. Res., 80, 2057, 1975.

Volland, H., A model of the magnetospheric electric convection field, $J$. Geophys. Res., 83, 2695-2699, 1978.

Wickwar, V. B. and W. Kofman, Dayside red auroras at very high latitudes: The importance of thermal excitation, Geophys. Res. Lett., 11, 923-926, 1984.

Zmuda, A. J., and J. C. Armstrong, The diurnal flow pattern of fieldaligned currents, J. Geophys. Res., 79, 4611, 1974.

Zmuda, A. J., J. H. Martin, and F. T. Heuring, Transverse magnetic disturbances at $1100 \mathrm{~km}$ in the auroral region, J. Geophys. Res., 71, $5033,1966$.

M. D. Bowline, R. W. Schunk, and J. J. Sojka, Center for Atmospheric and Space Sciences, UMC 4405, Utah State University, Logan, UT 84322.
(Received December 5, 1986; revised February 25, 1987; accepted February 10, 1987.) 